\title{
Effect of an immunomodulatory regimen for cancer prevention: A case report
}

\author{
WENBO WANG $^{1^{*}}$, WEI WANG ${ }^{2 *}$, BING LIU ${ }^{2}$, ZHUOYAO SUO $^{2}$, LIN ZHOU $^{2}$ and GUANGXIAN LIU ${ }^{2 *}$ \\ ${ }^{1}$ Chinese Medicine Department; ${ }^{2}$ Cancer Therapy Center, Affiliated Hospital of the Academy \\ of Military Medical Sciences, Beijing 100071, P.R. China
}

Received June 10, 2016; Accepted August 18, 2016

DOI: $10.3892 / \mathrm{mco} .2016 .1018$

\begin{abstract}
In the present case study, an immunomodulatory regimen for cancer prevention is reported. A patient with an abnormally high level of the tumor markers, carbohydrate antigen-724 (CA724), CA19-9 and carcinoembryonic antigen (CEA), although without any detectable tumor, was treated with an immunomodulatory therapy featuring an infusion of cytokine-induced autologous killer cells (CIKs) at the request of the patient. Following the therapy, the three tumor markers rapidly decreased to below the normal reference level, although there still were slight fluctuations within a narrow range frequently. The patient was monitored for 21 months to the present day and no abnormality was observed. The results indicated that this therapy may be applied as a novel strategy that is effective and reliable for cancer prevention. As there is no promising regimen for the prevention of malignancies to date, such a treatment may become a major cancer prophylactic regimen, particularly for patients who are at a high risk of cancer.
\end{abstract}

Correspondence to: Dr Guangxian Liu, Cancer Therapy Center, Affiliated Hospital of the Academy of Military Medical Sciences, Dongdajie 8, Beijing 100071, P.R. China

E-mail: liugx270@hotmail.com

${ }^{*}$ Contributed equally

Abbreviations: CIK, cytokine-induced autologous killer cell; $\mathrm{T}_{\text {reg }}$, regulatory $\mathrm{T}$ cell; $\mathrm{NK}$, natural killer cell; $\mathrm{HPV}$, human papillomavirus; HIV, human immunodeficiency virus; RT-qPCR, reverse transcription-quantitative polymerase chain reaction; IL, interleukin; IFN, interferon; CA, carbohydrate antigen-724; CEA, carcinoembryonic antigen; TGF- $\beta$, transforming growth factor- $\beta$; CTLA-4, cytotoxic T-lymphocyte-associated protein 4; PD-1, programmed cell death protein 1

Key words: cancer, cytokine-induced autologous killer cells, cytokine, cellular immunity, immunomodulation

\section{Introduction}

Although a great progression has been achieved in cancer therapy, as well as in cancer immunotherapy, including the moleculartargeting medicine and the recently developed 'immunocheckpoint' blocking strategy, which was considered as having opened up a new era in the fight against cancers, it remains a challenge for humans to cure cancer to date $(1,2)$. It is believed that early prevention is the most ideal strategy for combating cancers. However, with the exception of risk avoidance and early detection and treatment, a practical and promising regimen does not exist for the effective prevention of most malignancies to date, although over a century has passed since the 1890s, when investigations into, and clinical trials of, tumor vaccines and cancer immunotherapy were initiated (3-7). Previously, our group has reported a selective immunomodulation regimen for cancer immunotherapy, which comprised the downregulation of tumor-induced regulatory $\mathrm{T}$ cells $\left(\mathrm{T}_{\mathrm{reg}}\right)$ and upregulation of natural killer (NK) cells for maintaining the balance of cellular immunity in malignancies, termed 'immunoediting therapy' (8). As a novel regimen that focuses on dynamic monitoring, the timely elimination of tumor-induced immunosuppressive elements and maintaining the balance of cellular immunity, it is considered that it may become an essential treatment for patients with malignancies, and offer an improvement with respect to cancer prevention. In the present study, the results of a prophylactic treatment for a patient at a high risk of cancer are reported.

\section{Patients and methods}

Patient characteristics. A 78-year-old male patient, who did not smoke or drink alcohol and who had no family history of malignancy or other clear organ abnormalities, presented at the Affiliated Hospital of the Academy of Military Medical Sciences, Beijing, China. Initially, abnormally high levels of the tumor markers, carbohydrate antigen-724 (CA724), CA19-9 and carcinoembryonic antigen (CEA), were detected during a routine physical examination in February 2013. Since then, the patient has received Selenium pill and pollen pini., and the three tumor markers were monitored monthly until July 2014, but the three markers remained higher than normal, with levels recorded of 257-2,691 U/ml, 24.87-31.01 U/ml and 4.94-10.85 ng/ml, respectively. The patient received 
two positron emission tomography (PET) examinations on March 20, 2013 and April 9, 2014, respectively, and on neither occasion were any abnormalities identified. The patient refused to allow us to perform a further examination by gastrointestinal endoscopy, and was diagnosed as being at a high risk of cancer, with a recommendation for further observation and examination. On a rational basis, such a patient was suited for early cancer prophylactic therapy. At the request of the patient for further prophylactic treatment, he was administered the immunomodulatory therapy from July 2014, with two courses of cytokine injection and four courses of infusion of CIKs administered over an 8-month period, leading to February 2015 (Fig. 1A). The patient was further followed up until August 6, 2016 (data not shown). The most recent PET examination was performed in April 9, 2016, and everything appeared to be normal. The three markers were $4.9 \mathrm{U} / \mathrm{ml}, 44.66 \mathrm{U} / \mathrm{ml}$ and $5.16 \mathrm{ng} / \mathrm{ml}$ for CA724, CA19-9 and CEA, respectively (detected in December 2015), and $4.43 \mathrm{U} / \mathrm{ml}, 33.31 \mathrm{U} / \mathrm{ml}$ and $3.66 \mathrm{ng} / \mathrm{ml}$, respectively (detected in August 6,2016), without any other abnormalities.

Tumor marker detection. Serum CA724 (cat. no. 11776258112), CA19-9 (cat. no. 11776193122) and CEA (cat. no. 11731629322) were detected using an electrochemiluminescence immunoassay according to the manufacturer's specifications. A Roche $\operatorname{cobas}^{\circledR} 6000$ electrochemical luminescence instrument was employed in this assay.

Analysis of the cellular immunity. To monitor changes in cellular immunity, T-and B-lymphocytes, NKs, $\mathrm{T}_{\text {reg }}$ and other major lymphocyte subpopulations in the peripheral blood were analyzed prior to and following each course of treatment using four-color flow cytometric analysis (EPICS XL; Beckman Coulter Inc., Brea, CA, USA). Fluorescein isothiocyanate-, phycoerythrin (PE)-Cy5-, PerCP-, allophycocyanin (APC)- and PE-Texas Red (ECD)-conjugated antibodies against CD45 (20 $\mu 1 /$ test, cat. no. 347464), CD3 (5 $\mu \mathrm{l} /$ test, cat. no. 340440), CD4 (20 $\mu \mathrm{l} /$ test, cat. no. 340133), CD8 (20 $\mu \mathrm{l} /$ test, cat. no. 340046), CD56 (20 $\mu \mathrm{l} /$ test, cat. no. 340636), CD19 (5 $\mu 1 /$ test, cat. no. 340437), CD25 (5 $\mu 1 /$ test, cat. no. 340939), CD28 (20 $\mu 1 /$ test, cat. no. 348047), human leukocyte antigen-antigen D-related (HLA-DR) $20 \mu \mathrm{l} /$ test, cat. no. 552764), CD45RA (20 $\mu \mathrm{l} /$ test, cat. no. 347723) and CD45RO (20 $\mu \mathrm{l} /$ test, cat. no. 347967) were purchased from BD Biosciences, Franklin Lakes, NJ, USA. Cells were labeled according to the manufacturer's protocols. The data were initially collected on day -3 to day 0 prior to the treatment. The posttreatment data were collected immediately following treatment, in most cases, on the last day of a course.

Analysis of gene expression. The expression of eight genes [interferon (IFN)-r, CD25, CD28, interleukin (IL)-10, transforming growth factor- $\beta$ (TGF- $\beta$ ), Foxp3, cytotoxic T-lymphocyte-associated protein 4 (CTLA-4) and programmed cell death protein 1 (PD-1)] with immuneactivated or immunosuppressive functions was analyzed using a reverse transcription-quantitative polymerase chain reaction (RT-qPCR)-based method (cat. no. 201411; Beijing Mo Li Tai Bio-Technology, Inc., Beijing, China). Briefly, lymphocytes were collected from the peripheral blood of the patient by centrifugation at $400 \mathrm{x}$ g for $5 \mathrm{~min}$ at room temperature, and subsequently the cells collected were mixed with red blood cell lysing solution (cat. no. 21510; Beijing Dong Fang Hua Hui Biomedical Technology, Inc., Beijing, China) at a ratio of 1:9 for $10 \mathrm{~min}$, prior to centrifuging at $400 \mathrm{x} \mathrm{g}$ for $5 \mathrm{~min}$ at room temperature in order to obtain the lymphocytes. Total RNA was isolated using a standard RNA isolation procedure, according to the manufacturer's protocol (cat no. 15596-026; Thermo Fisher Scientific, Inc, Beijing, China). The RNA was subsequently reverse-transcribed into complementary (c)DNA using a reverse-transcription system (cat. no. A3500; Promega Corp., Madison, WI, USA). Reagents were used and the RT protocol was performed according to the A3500 instruction. Briefly, total RNA and Oligo(dT) $(0.5 \mu \mathrm{g})$ were incubated at $70^{\circ} \mathrm{C}$ for $10 \mathrm{~min}$, and subsequently placed on ice. The $20 \mu \mathrm{l}$ reaction was prepared by adding the following reagents: $25 \mathrm{mM} \mathrm{MgCl} 2$, $4 \mu \mathrm{l}$; Reverse Transcription 10X buffer, $2 \mu \mathrm{l} ; 10 \mathrm{mM}$ dNTP mixture, $2 \mu \mathrm{l}$; Recombinant RNasin ${ }^{\circledR}$ Ribonuclease Inhibitor, $0.5 \mu \mathrm{l}$; AMV Reverse Transcriptase, $15 \mathrm{U}$; and nuclease-free water to a final volume of $20 \mu \mathrm{l}$. The samples were heated at $95^{\circ} \mathrm{C}$ for $5 \mathrm{~min}$, and subsequently the cDNA synthesis reaction was incubated at $42^{\circ} \mathrm{C}$ for up to $60 \mathrm{~min}$ for longer and/or more abundant transcripts. Forty cycles were applied in the program for the PCR reaction. qPCR assay of the expression of the eight genes was performed using the Applied Biosystems ${ }^{\circledR}$ 7500 Real-Time PCR System (Applied Biosystems Life Technologies, Beijing, China).

Expansion of autologous cytokine-induced killer cells (CIK) in vitro. Two complete aphereses of autologous peripheral blood mononuclear cells (PBMCs) were performed using a CS3000S cell separator (Baxter, Deerfield, IL, USA). The cell numbers apheresed were $1.28 \times 10^{9}$ and $1.47 \times 10^{9}$, respectively, and two aliquots were separated and cryopreserved from the second apheresis for later treatment. CIKs were prepared as previously described (8). Briefly, newly apheresed or thawed PBMCs were cultured in TexMACS ${ }^{\mathrm{TM}}$ GMP Medium (Miltenyi Biotec, Bergisch Gladbach, Germany) with 1,000 U/ml IFN- $\gamma$ (Beijing ShuangLu Pharmaceutical Co., Ltd., Beijing, China) added on day 0, $50 \mathrm{ng} / \mathrm{ml}$ antiCD3 (Hui Xin Qing Yuan Biotech Co., Ltd., Beijing, China) added on day 1, and $500 \mathrm{U} / \mathrm{ml}$ recombinant human IL2 included in the medium from day 1 onwards. From days 10 to 21 , the cells were harvested on every other day for infusion.

Statistical analysis. Statistical analyses of the data were performed using GraphPad Prism software (San Diego, CA, USA).

\section{Case report}

Clinical protocol and treatment arrangement. The protocol was approved by the Human Ethics Committee of the Affiliated Hospital of the Academy of Military Medical Sciences, Beijing, China, and was conducted in accordance with the Declaration of Helsinki. The patient received immunomodulatory therapy and an infusion of CIKs after informed consent, and his cellular immunity was monitored prior to and following each course of treatment (8). IFN- $\alpha$-1b (Beijing Tri-Prime Gene Inc. Beijing, China), IL-2 [Beijing Shuanglu (SL) Pharmaceutical 
$\mathbf{A}$

Immunomodulatory therapy

CIK infusion

IFN- $\alpha$-1b \& Thymalfasin

$\mathbf{B}$
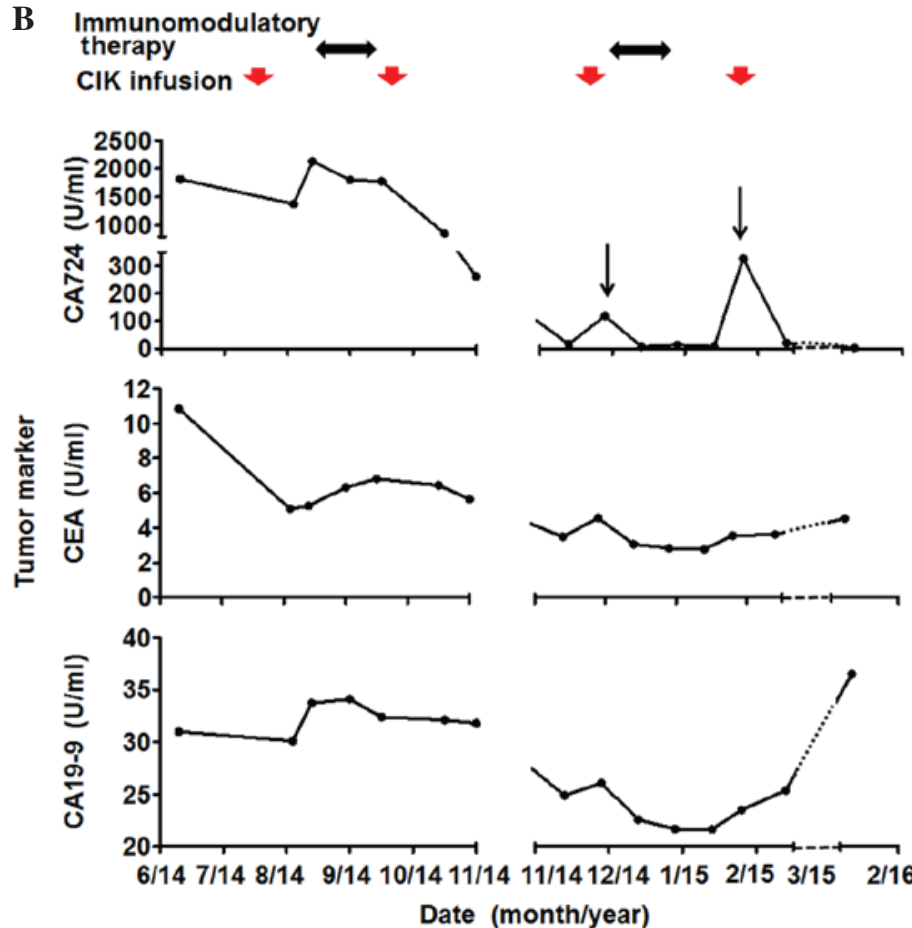

C

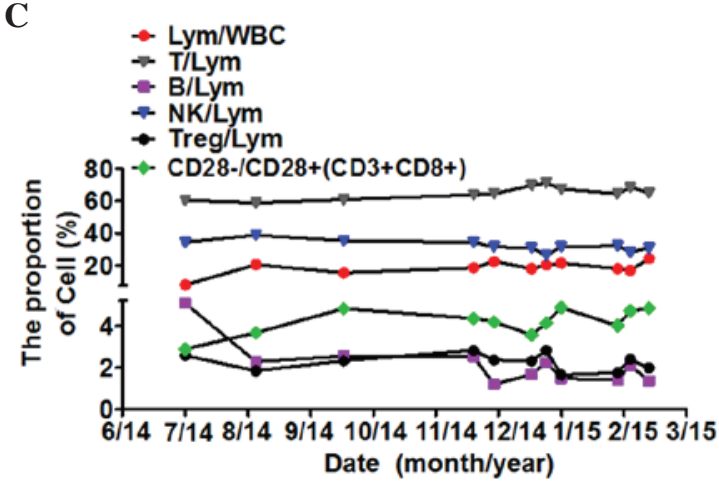

D

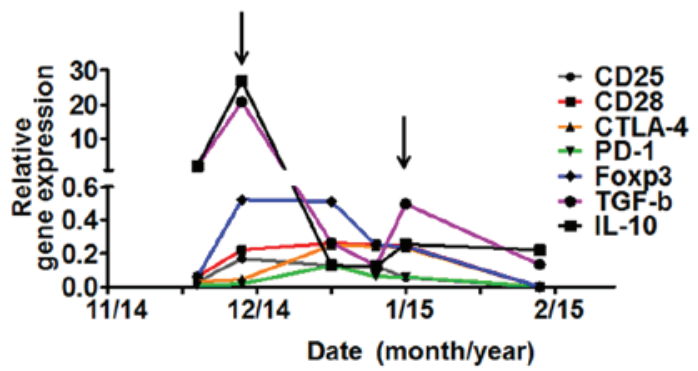

Figure 1. A prophylactic treatment for a patient with a high risk of cancer. (A) A model of the therapeutic regimen. The patient received a combinatorial treatment with CIKs and cytokines. (B) The shifts that occurred in the levels of serum CA724, CA19-9 and CEA during the therapy are shown. The serum expression levels of CA724, CA19-9 and CEA were detected using an enzymelinked immunosorbence assay. (C) Analysis of cellular immunity. Flow cytometry was performed to analyze the Lym proportions (as percentages of the overall population) for $\mathrm{T}, \mathrm{B}, \mathrm{NK}, \mathrm{T}_{\mathrm{reg}}$ and other cell types in the peripheral blood. (D) Gene expression of molecules associated with the processes of immune activation or immunosuppression, including IFN-r, CD25, CD28, IL-10, TGF- $\beta$, Foxp3, CTLA-4 and PD-1, which were detected using an RT-qPCR method. IL, interleukin; IFN, interferon; CIK, cytokine-induced autologous killer cell; CA, carbohydrate antigen-724; CEA, carcinoembryonic antigen; Lym, lymphocyte; WBC, white blood cell; TGF- $\beta$, transforming growth factor- $\beta$; CTLA-4, cytotoxic Tlymphocyteassociated protein 4; PD-1, programmed cell death protein 1.

Inc., Beijing, China] and thymalfasin (Hainan Zhonghe Pharmaceutical Co., Ltd., Hainan, China) were administered, with doses of $300 \mathrm{~m}$-units, $100 \mathrm{~m}$-units and $1 \mathrm{mg}$, respectively, for each injection. The immunomodulatory therapy was designed according to the status of the cellular immunity (8). As shown in Fig. 1A, the patient first received a CIK infusion intravenously (six injections in 6 days) with a total number of $1.29 \times 10^{9}$ cells; subsequently, he received immunomodulatory therapy with IL- 2 and IFN- $\alpha-1 b$ (10 injections per course), administered respectively by intracutaneous injection each day on alternating days, predominantly in order to downregulate $\mathrm{T}_{\text {reg }}$, and upregulate $\mathrm{NK}$, function (8). The second and third courses of CIK infusion were performed in the third and the fourth months, respectively, each with six injections over the course of 6 days and a total number of $1.53 \times 10^{9}$ and $5.55 \times 10^{8}$ cells, respectively. The second immunomodulatory therapy was provided in the fifth month immediately following the third course of CIK infusion with thymalfasin and IFN- $\alpha-1 b$; the former was administered by 15 injections intramuscularly, the latter by 12 injections intracutaneously, administered respectively on alternating days, predominantly for the up-modulation of CD4+ T-cells and to downregulate $\mathrm{T}_{\text {reg }}$ and the ratio of $\mathrm{CD} 8^{+} \mathrm{CD} 28 / \mathrm{CD} 8^{+} \mathrm{CD} 28^{+}$cells. The fourth course of CIK infusion was performed in the seventh month, with six injections over the course of 6 days and a total number of $7.89 \times 10^{8}$ cells.

Effective cancer prophylactic results, and the experimental findings. As shown in Fig. 1B, the levels of the three tumor markers in serum decreased rapidly following the therapy and were maintained at under, or sometimes around, the reference level, though there were several slight up-waves during the follow-up period. During the therapy, the lymphocyte subpopulations, e.g. of T-, B-, NK and $\mathrm{T}_{\text {reg }}$ lymphocytes and others, in peripheral blood were also monitored simulta- 
neously (Fig. 1C). The data revealed that the $\mathrm{B}$ and $\mathrm{T}_{\text {reg }}$ subpopulations experienced a marked down-modulation following the first course of treatment, whereas the others were maintained at a moderate level over the course of the short treatment time-span. Furthermore, the expression of several molecule markers with immune-activated or immunosuppressive functions, including IFN-r, CD25, CD28, IL-10, TGF- $\beta$, Foxp3, CTLA- 4 and PD-1, were also analyzed by an RT-qPCR method at the latter time points (Fig. 1D). The results demonstrated that, accompanying the partial restoration in the level of CA724 from 16 to 218 , the subsequent decrease to 6 , the second partial restoration from 7.74 to 326.3 , and the second decrease to $20.19 \mathrm{U} / \mathrm{ml}$ following further treatments, the expression of the immune-activated and immunosuppressive molecules also markedly changed. Notably, the levels of all the molecules, with the exception of CTLA-4 and PD-1, were upregulated at the time point that coincided with the first partial restoration in the level of CA724, after which CTLA-4 and PD-1 were also markedly upregulated, CD28, Foxp3 and CD25 were maintained at a high level, and TGF- $\beta$ and IL-10 dropped markedly following the first course of the treatment. At the time point coinciding with the second partial restoration in the level of CA724, CD28 and CTLA-4 were still maintained at a high level, and TGF- $\beta$ and IL-10 were markedly upregulated, whereas the others were maintained at their downregulated levels. All the molecules were downmodulated following a further course of treatment. IFN-r was also monitored, although it was undetectable, whereas it was detected at a very low level following stimulation with phorbol 12-myristate 13-acetate (PMA), indicating its primary expression level was too low to be detected by the RT-qPCR protocol (data not shown). Cytometric analysis revealed that $10 \%$ of the $\mathrm{CD}^{+}$population was expressing IFN-r in the blood at the time point of December 2014.

\section{Discussion}

To a certain extent, malignancy may be considered as an 'immunosurveillance-defective' disease. Due to the disabled function of immunosurveillance, mutated and malignant-transformed cells are able to survive and grow, finally develop into the malignant disease state, and also to accelerated recurrence and metastasis in patients (9-12). In this sense, a normal or balanced function of the immune system, particularly concerning cellular immunity, is a key factor in the prevention of malignancies in humans $(13,14)$. In malignant patients, normal cellular immunity is often disrupted severely, including the function of the T-, B- and NK lymphocytes, as well as the dynamic balance between immunoactivation and immunosuppression (10,15-17). Therefore, the monitoring, and a timely modulation of, dysregulated cellular immunity in order to maintain potent immunosurveillance may become a promising cancer prophylactic regimen for individuals at a high risk of cancer, and offer a basic treatment for malignancies $(10,18)$. It has been intensively researched that cancer development is a long-term process, and that the majority of the cellular events occur during the pre-clinical stage (19). In addition to a family background of inherited cancer and the positive detection of oncogenes, various signs of cancer risk, including abnormally elevated tumor markers, severe infec- tion of HPV or human immunodeficiency virus (HIV) with a family history of cancer, familial adenomatous polyposis, severe atrophic gastritis or severe Helicobacter pylori infection, and so forth, may be identified through a routine physical examination. Cancer prevention is rational in such a situation as this, and the advent of a promising cancer prophylactic technology is urgently required for such a population.

In the present case report, a patient presenting with abnormally high levels of the tumor markers CA724, CA19-9 and CEA received such a prophylactic regimen without any other treatment. The results demonstrated that the levels of the three tumor markers declined rapidly following the therapy, and were maintained at around normal levels for 21 months from November 2014 to the present day, suggesting the effectiveness of the regimen.

The exact antitumor mechanism that underpins this process has yet to be elucidated. It is considered that the selective immunomodulation and long-term dynamic maintenance of a balanced cellular immune function by providing the immunoediting therapy exerted a key role; that is, a normal immune function could also exert a potent therapeutic antitumor effect, as the levels of the three tumor markers began to fall immediately at the beginning of the therapy, and continued to fall afterwards. Based on an analysis of the cytometric results of the lymphocyte subpopulations and the application of RT-qPCR for an evaluation of the expression of several immunoregulatory molecules with immune-activated or immunosuppressive functions in the latter time points of the treatment, it appears that the downregulation of the $\mathrm{B}$ and $\mathrm{T}_{\text {reg }}$ lymphocyte subpopulations and of TGF- $\beta$ and IL-10 expression, as well as the maintenance of Th1 cellular immunity, made significant contributions, although the expression of IFN-r was not upregulated. Notably, although all the functional molecules (with the exception of IFN-r) were upregulated at the time point where the first partial restoration in the level of CA724 occurred, at the time point coinciding with the second partial restoration in the CA724 level, only the expression of TGF- $\beta$ and IL-10 altered in accordance with the change in the CA724 level, indicating in part that, besides the IFN-r mechanism, the relative downregulation of immunosuppressive factors may also give rise to effective antitumor effects, which may be meaningful in terms of cancer immunotherapy. The upregulation of CTLA-4 and PD-1, as well as the subsequent maintenance of their elevated levels, was considered to have occurred as a consequence of a feedback effect resulting from the therapy. As they did not decline in accordance with the decreases subsequently observed for the other tumor markers, the role they exerted in terms of modulation of the antitumor effect, in comparison with factors such as TGF- $\beta$ and IL-10, has yet to be fully elucidated. Furthermore, the observed partial restoration in the levels of CA724 itself was also potentially of significance. It appears that, during the treatment and under conditions of immune pressure, the mechanism could have been that the expression of CA724 was inhibited even though the CA724-expressing cells were not killed, indicating the possibility of reverse transformation of malignant-transformed cells in the early-transformation stage under immune pressure. Further investigation is required to elucidate the exact antitumor mechanism underpinning this novel regimen. 
In conclusion, the present case report has introduced and evaluated an immunomodulatory regimen for cancer prevention, which may be particularly of benefit for those who are at high risk of cancer.

\section{Acknowledgements}

The present study was supported by the National Natural Science Foundation (no. 81372251).

\section{References}

1. Cataldo VD, Gibbons DL, Pérez-Soler R and Quintás-Cardama A: Treatment of non-small-cell lung cancer with erlotinib or gefitinib. N Engl J Med 364: 947-955, 2011.

2. Hodi FS, O'Day SJ, McDermott DF, Weber RW, Sosman JA, Haanen JB, Gonzalez R, Robert C, Schadendorf D, Hassel JC, et al: Improved survival with ipilimumab in patients with metastatic melanoma. N Engl J Med 363: 711-723, 2010.

3. Coley WB: The treatment of malignant tumors by repeated inoculations of erysipelas. With a report of ten original cases. 1893. Clin Orthop Relat Res 262: 3-11, 1991.

4. Rosenberg SA, Lotze MT, Muul LM, Leitman S, Chang AE, Ettinghausen SE, Matory YL, Skibber JM, Shiloni E, Vetto JT, et al: Observations on the systemic administration of autologous lymphokine-activated killer cells and recombinant interleukin-2 to patients with metastatic cancer. N Engl J Med 313: 1485-1492, 1985.

5. Atzpodien J, Kirchner H, Jonas U, Bergmann L, Schott H, Heynemann H, Fornara P, Loening SA, Roigas J, Müller SC, et al; Prospectively Randomized Trial of the German Cooperative Renal Carcinoma Chemoimmunotherapy Group (DGCIN): Interleukin-2- and interferon alfa-2a-based immunochemotherapy in advanced renal cell carcinoma: A Prospectively Randomized Trial of the German Cooperative Renal Carcinoma Chemoimmunotherapy Group (DGCIN). J Clin Oncol 22: 1188-1194, 2004.

6. Schuler G, Schuler-Thurner B and Steinman RM: The use of dendritic cells in cancer immunotherapy. Curr Opin Immunol 15: 138-147, 2003.

7. Finn OJ: Cancer vaccines: Between the idea and the reality. Nat Rev Immunol 3: 630-641, 2003.
8. Liu G, Yang W, Guo M, Liu X, Huang N, Li D, Jiang Z, Yang W, Zhang W, Su H, et al: Effective modulation of CD4(+)CD25 (+high) regulatory $\mathrm{T}$ and $\mathrm{NK}$ cells in malignant patients by combination of interferon- $\alpha$ and interleukin-2. Cancer Immunol Immunother 61: 2357-2366, 2012.

9. Dunn GP, Bruce AT, Ikeda H, Old LJ and Schreiber RD: Cancer immunoediting: From immunosurveillance to tumor escape. Nat Immunol 3: 991-998, 2002.

10. Rabinovich GA, Gabrilovich D and Sotomayor EM: Immunosuppressive strategies that are mediated by tumor cells. Annu Rev Immunol 25: 267-296, 2007.

11. Valzasina B, Piconese S, Guiducci C and Colombo MP: Tumor-induced expansion of regulatory $\mathrm{T}$ cells by conversion of CD4+CD25- lymphocytes is thymus and proliferation independent. Cancer Res 66: 4488-4495, 2006.

12. Jarnicki AG, Lysaght J, Todryk S and Mills KH: Suppression of antitumor immunity by IL-10 and TGF-beta-producing T cells infiltrating the growing tumor: Influence of tumor environment on the induction of $\mathrm{CD} 4+$ and $\mathrm{CD} 8+$ regulatory $\mathrm{T}$ cells. J Immunol 177: 896-904, 2006.

13. Vesely MD, Kershaw MH, Schreiber RD and Smyth MJ: Natural innate and adaptive immunity to cancer. Annu Rev Immunol 29: 235-271, 2011.

14. Shankaran V, Ikeda H, Bruce AT, White JM, Swanson PE, Old LJ and Schreiber RD: IFNgamma and lymphocytes prevent primary tumour development and shape tumour immunogenicity. Nature 410: 1107-1111, 2001.

15. Ostrand-Rosenberg S and Sinha P: Myeloid-derived suppressor cells: Linking inflammation and cancer. J Immunol 182: 4499-4506, 2009.

16. Zhang L, Conejo-Garcia JR, Katsaros D, Gimotty PA, Massobrio M, Regnani G, Makrigiannakis A, Gray H, Schlienger K, Liebman MN, et al: Intratumoral $\mathrm{T}$ cells, recurrence, and survival in epithelial ovarian cancer. $\mathrm{N}$ Engl $\mathrm{J}$ Med 348: 203-213, 2003.

17. Trzonkowski P, Szmit E, Myśliwska J, Dobyszuk A and Myśliwski A: CD4+CD25+ T regulatory cells inhibit cytotoxic activity of T CD8+ and NK lymphocytes in the direct cell-to-cell interaction. Clin Immunol 112: 258-267, 2004.

18. Gattinoni L, Powell DJ Jr, Rosenberg SA and Restifo NP Adoptive immunotherapy for cancer: Building on success. Nat Rev Immunol 6: 383-393, 2006.

19. Loeb LA, Loeb KR and Anderson JP: Multiple mutations and cancer. Proc Natl Acad Sci USA 100: 776-781, 2003. 